\title{
Lie Group Method of the Diffusion Equations
}

\author{
Jian-Qiang Sun, ${ }^{1}$ Rong-Fang Huang, ${ }^{1}$ Xiao-Yan $\mathrm{Gu}^{2}{ }^{2}$ and Ling $\mathrm{Yu}^{1}$ \\ ${ }^{1}$ Department of Mathematics, College of Information Science and Technology, Hainan University, Haikou 570228, China \\ ${ }^{2}$ Department of Physics, East China University of Science and Technology, Shanghai 200237, China
}

Correspondence should be addressed to Jian-Qiang Sun; sunjq123@qq.com

Received 1 January 2014; Accepted 7 April 2014; Published 29 April 2014

Academic Editor: Shi-Hai Dong

Copyright (C) 2014 Jian-Qiang Sun et al. This is an open access article distributed under the Creative Commons Attribution License, which permits unrestricted use, distribution, and reproduction in any medium, provided the original work is properly cited.

\begin{abstract}
The diffusion equation is discretized in spacial direction and transformed into the ordinary differential equations. The ordinary differential equations are solved by Lie group method and the explicit Runge-Kutta method. Numerical results showed that Lie group method is more stable than the corresponding explicit Runge-Kutta method.
\end{abstract}

\section{Introduction}

The subject of solving stiff system has been well studied in the literature, including linearly implicit methods, semi-implicit methods, time-splitting methods, projection methods, multiscale methods, integrating factor methods, the exponential time difference (ETD) methods, and Lie group methods [112]. Lie group methods have been developed by MuntheKaas, Iserles, Nørsett, and their collaborators. In general, if a Lie group $G$ acts freely and transitively on a manifold $M$, then a differential equation on $M$ uniquely determines a differential equation on $G$. Thus, replacing the original differential on $M$ by the equivalent differential equation on Lie algebra $g$ allows one to apply appropriate Lie group integrators. There are many Lie group methods, such as Mangus method, RKMK method, and Fer method. Lie group methods can preserve the numerical solutions of the differential equations on the same manifolds, which have good stability and the same accuracy as the classical numerical methods $[1,2,11,13$, 14]. In the paper, the exponential integrator method of the general nonlinear differential equations is proposed based on the idea of Lie group methods on manifolds and applied to the diffusion equations.

The paper is organized as follows: in Section 2, the Lie group method and the exponential time difference method are introduced. The exponential integrator method for the general nonlinear differential equations is proposed. In Section 3, the quasilinear diffusion equation is solved by the explicit exponential integrator method and the explicit
Runge-Kutta methods. In Section 4, the Allen-Cahn equation is solved by the explicit exponential integrator method and the explicit Runge-Kutta methods. At last, some conclusions are obtained.

\section{Exponential Integrator}

Suppose that $M$ is a manifold. There exist a Lie algebra $g$ with a Lie bracket $[\cdot, \cdot]$, a (left) Lie algebra action $\lambda: g \times M \rightarrow M$, and a function $f: R \times M \rightarrow g$ such that the equation for $y(t) \in M$ can be written as

$$
y^{\prime}=\left(\lambda_{*} f(t, y)\right)(y), \quad y(0)=p,
$$

where

$$
\lambda_{*}: g \longrightarrow \aleph(M)
$$

is a homomorphism. The left algebra action $\lambda: g \times M \rightarrow M$ assigns to the arguments $v \in g$ and $y_{0}(x) \in M$ the solution of

$$
y_{t}=\left(\lambda_{*} v\right)(y)
$$

at time $t=1$ with the initial condition $y_{0}(x)$ and some boundary conditions. There is freedom to choose the function $f$, the corresponding differential equation in the Lie algebra

$$
\frac{d v}{d t}=\operatorname{dexpinv}\left(v, \mathrm{f}\left(\mathrm{t}, \lambda\left(v, \mathrm{y}_{0}\right)\right)\right), \quad v(0)=\mathrm{O} .
$$


Lie algebra is chosen as functions corresponding to simpler vector fields on $M$ which are easy to exponentiate. The correspondence between the Lie algebra and the vector fields on $M$ will be one to one. The Runge-Kutta method is applied to solve (4). It is the well-known RKMK method [1].

Let $a_{i, j}$ and $b_{j}$ be the coefficients of an $s$-stage, $q$ order classical Runge-Kutta method, and let $c_{i}=\sum_{j=1}^{s} a_{i, j}$. The algorithm integrates (1) from $t=0$ to $t=h$ :

Set $y_{0}=p$.

For $i=1,2, \ldots, s$

$$
u_{i}=h \sum_{j=1}^{s} a_{i, j} \tilde{k}_{j}, \quad k_{i}=f\left(h c_{i}, \lambda\left(u_{i}, y_{0}\right)\right)
$$

$$
\tilde{k}_{i}=\operatorname{dexpinv}\left(\mathrm{u}_{\mathrm{i}}, \mathrm{k}_{\mathrm{i}}, \mathrm{q}\right), \quad \mathrm{q}=\text { the order of RK method. }
$$

End

$$
\begin{gathered}
v=h \sum_{j=1}^{s} b_{j} \widetilde{k}_{j}, \\
y_{1}=\lambda\left(v, y_{0}\right),
\end{gathered}
$$

where

$$
\operatorname{dexpinv}(\mathrm{u}, v, \mathrm{q})=\sum_{\mathrm{k} !}^{\mathrm{q}-1} \frac{\mathrm{B}_{\mathrm{k}}}{\mathrm{k} !} \mathrm{ad}_{\mathrm{u}}^{\mathrm{k}}(v)
$$

$a d_{u}^{0}(v)=v, a d_{u}^{1}(v)=[u, v]$ and $a d_{u}^{k}(v)=a d_{u}\left(a d_{u}^{k-1}(v)\right)$. The number $q$ denotes the order of the underlying Runge-Kutta method and $B_{k}$ is the $k$ th Bernoulli number [2].

A system of ordinary differential equations is often obtained

$$
y_{t}=L y+N(y, t), \quad y \in R^{n},
$$

where $L \in R^{n \times n}$. The ETD method can be described in the context of solving (8). Integrating the equation over a single time step from $t=t_{n}$ to $t_{n+1}=t_{n}+h$, we can get

$$
\begin{aligned}
y\left(t_{n+1}\right)= & e^{L h} y\left(t_{n}\right) \\
& +e^{L h} \int_{0}^{h} e^{-L s} N\left(y\left(t_{n}+s\right), t_{n}+s\right) d s .
\end{aligned}
$$

Equation (9) is exact, and the various ETD schemes come from the approximation to the integral. Suppose $y_{n}$ is the approximation to $y\left(t_{n}\right)$, and the first order ETD method is given by

$$
y_{n+1}=e^{L h} y_{n}+L^{-1}\left(e^{L h}-I\right) N\left(y_{n}, t_{n}\right) .
$$

Higher order ETD schemes can also be found [7, 9, 10]. Some preliminary numerical studies conducted by a group of material scientists at the Penn State University have indicated that the higher order ETD schemes can be several orders of magnitudes faster than the low-order semi-implicit methods in some simulations of microstructure evolution [8].
In essence, for nonlinear time dependent equations, the ETD scheme provides a systematic coupling of the explicit treatment of nonlinearities and the implicit and possibly exact integration of the stiff linear part of the equation, while achieving high accuracy and maintaining good stability. The exponential integrator of (8) is constructed based on the idea of Lie group methods on manifolds. In general, (8) can be written as

$$
y^{\prime}=A(t, y) y, \quad y(0)=y_{0}, \quad y \in R^{n},
$$

where $A: R \times R^{n} \rightarrow R^{n \times n}$. In addition, $A$ should be locally Lipschitz function (to ensure existence and uniqueness). The classical fourth order explicit RK scheme of (11) is

$$
\begin{aligned}
V_{1} & =Y_{n}, \quad K_{1}=A\left(t_{n}, V_{1}\right) V_{1}, \\
V_{2} & =Y_{n}+\frac{1}{2} h K_{1}, \quad K_{2}=A\left(t_{n}+\frac{1}{2} h, V_{2}\right) V_{2}, \\
V_{3} & =Y_{n}+\frac{1}{2} h K_{2}, \quad K_{3}=A\left(t_{n}+\frac{1}{2} h, V_{3}\right) V_{3}, \\
V_{4} & =Y_{n}+h K_{3}, \quad K_{4}=A\left(t_{n}+h, V_{4}\right) V_{4}, \\
Y_{n+1} & =Y_{n}+h\left(\frac{1}{6} K_{1}+\frac{1}{3} K_{2}+\frac{1}{3} K_{3}+\frac{1}{6} K_{4}\right) .
\end{aligned}
$$

According to a classical result of Hausdorff, the solution of (11) is

$$
y(t)=e^{X(t)} y(0),
$$

$X(t): R \rightarrow R^{n \times n}$ is the solution of the initial value problem

$$
X(t)^{\prime}=d \exp _{X(t)}^{-1} A(t, Y), \quad X\left(t_{0}\right)=O,
$$

where

$$
\begin{aligned}
d \exp _{X}^{-1}(A) & =A-\frac{1}{2}[X, A]+\frac{1}{12}[X,[X, A]]+\cdots \\
& =\sum_{j=0}^{\infty} \frac{B}{p !} a d_{X}^{p} A,
\end{aligned}
$$

$a d_{X}^{k} A=\left[X, a d_{X}^{k-1} A\right]$, and $[X, A]=X A-A X$. Taking $p$ as the corresponding number and applying the Runge-Kutta method to (14), we can get the numerical solution of (14). So the numerical solution of (11) is obtained. The corresponding $p$ order exponential integrator of (11) is

$$
\begin{aligned}
X_{k} & =\sum_{l=1}^{p} a_{k, l} F_{l}, \\
F_{k} & =d \exp _{X}^{-1}\left(A_{k}, p\right), \\
A_{k} & =h A\left(t_{n}+c_{k} h, \exp \left(X_{k}\right) y_{n}\right), \\
X & =\sum_{k=1}^{p} F_{k}, \\
y_{n+1} & =\exp (X) y_{n}
\end{aligned}
$$


for $n \in N$ and it is explicit provided that the underlying RK scheme is explicit. $d \exp _{X}^{-1}\left(A_{k}, p\right)$ is the $p$ order truncation error of the $d \exp _{X}^{-1}\left(A_{k}\right)$. An explicit fourth order exponential integrator of (11) is as follows:

$$
\begin{aligned}
V_{1} & =O, \quad K_{1}=A\left(t_{n}, y_{n}\right), \\
V_{2} & =\frac{1}{2} h K_{1}, \\
K_{2} & =A\left(t_{n}+\frac{1}{2} h, e^{V_{2}} y_{n}\right)-\frac{1}{2}\left[V_{2}, A\left(t_{n}+\frac{1}{2} h, e^{V_{2}} y_{n}\right)\right], \\
V_{3} & =\frac{1}{2} h K_{2}, \\
K_{3} & =A\left(t_{n}+\frac{1}{2} h, e^{V_{3}} y_{n}\right)-\frac{1}{2}\left[V_{3}, A\left(t_{n}+\frac{1}{2} h, e^{V_{3}} y_{n}\right)\right], \\
V_{4} & =h K_{3}, \\
K_{4} & =A\left(t_{n}+h, e^{V_{4}} y_{n}\right)-\frac{1}{2}\left[V_{4}, A\left(t_{n}+h, e^{V_{4}} y_{n}\right)\right], \\
\Delta & =h\left(\frac{1}{6} K_{1}+\frac{1}{3} K_{2}+\frac{1}{3} K_{3}+\frac{1}{6} K_{4}\right), \\
y_{n+1} & =e^{\Delta} y_{n} .
\end{aligned}
$$

As the Lie group method on manifolds, the exponential integrator method of the general nonlinear differential equation has the same accuracy as the corresponding Runge-Kutta methods. In the subsequent sections, the fourth order explicit exponential integrator (EEI) method and the corresponding explicit Runge-Kutta (ERK) method are applied to the diffusion equations.

\section{Numerical Experiments I: The Quasilinear Convection Diffusion Equation}

To the quasilinear convection diffusion equation

$$
\frac{\partial u}{\partial t}+u \frac{\partial u}{\partial x}=\frac{1}{\operatorname{Re}} \frac{\partial^{2} u}{\partial x}
$$

the equation is the one-dimensional quasilinear parabolic differential equation, which is known as Burgers' equation, where $\operatorname{Re}>0$ is a constant representing the kinematic viscosity of the fluid. Burgers' equation first appeared in a paper by Bateman and he gave a special solution of (18). In remarkable series of papers from 1939 to 1965, Burgers' investigated various aspects of turbulence and he used it as a model in studies of turbulence. Cole studied the general properties of Burgers' equation and outlined some of its various applications [15-17]. With the initial condition $u(x, 0)=$ $\sin (\pi x), 0 \leq x \leq 1$, and $t>0$, the exact solution of (18) is

$$
\begin{array}{r}
u(x, t) \\
=\frac{4 \pi}{\operatorname{Re}}\left(\left(\sum_{n=1}^{\infty} e^{-n^{2} \pi^{2} t / \operatorname{Re}} n I_{n}\left(\frac{\mathrm{Re}}{2 \pi}\right) \sin (n \pi x)\right)\right. \\
\times\left(I_{0}\left(\frac{\mathrm{Re}}{2 \pi}\right)+2 \sum_{n=1}^{\infty} e^{-n^{2} \pi^{2} t / \operatorname{Re}}\right. \\
\left.\left.\quad \times I_{n}\left(\frac{\operatorname{Re}}{2 \pi}\right) \cos (n \pi x)\right)^{-1}\right),
\end{array}
$$

and $I_{n}(x)$ is the Bessel function. Recently some numerical methods of Burgers' equation have been proposed [15-17].

Burgers' equation can be transformed into the nonlinear ordinary differential equation by discretization in the spacial direction. The spacial derivative of Burger' equation can be discretized as follows: $\left.(\partial u / \partial x)\right|_{x=x_{i}}=\left(\left(u_{i+1}-u_{i-1}\right) / 2 \Delta x\right)$. $\left.\left(\partial^{2} u / \partial^{2} x\right)\right|_{x=x_{i}}=\left(\left(u_{i+1}-2 u_{i}+u_{i-1}\right) /(\Delta x)^{2}\right)$. So the variable $u_{i}=u\left(x_{i}, t\right)$ can satisfy

$$
\frac{\partial u_{i}}{d t}+u_{i} \frac{\left(u_{i+1}-u_{i-1}\right)}{2 \Delta x}=\frac{1}{\operatorname{Re}} \frac{u_{i+1}-2 u_{i}+u_{i-1}}{(\Delta x)^{2}} .
$$

Supposing that $U=\left(u_{1}, u_{2}, \ldots, u_{N-1}\right),(20)$ can be written as

$$
\begin{gathered}
\frac{d U}{d t}=B(U) U \\
B(U)=\frac{\eta}{(\Delta x)^{2}}\left(\begin{array}{cccccc}
-2 & 1 & 0 & \cdots & 0 & 0 \\
1 & -2 & 1 & \cdots & 0 & 0 \\
\vdots & \vdots & \vdots & \ddots & \vdots & \vdots \\
0 & 0 & 0 & \cdots & -2 & 1 \\
0 & 0 & 0 & \cdots & 1 & -2 \\
& & & & &
\end{array}\right) \\
+\mu\left(\begin{array}{cccccc}
u_{2} & 0 & 0 & \cdots & 0 & 0 \\
0 & c_{2} & 0 & \cdots & 0 & 0 \\
\vdots & \vdots & \vdots & \ddots & \vdots & \vdots \\
0 & 0 & 0 & \cdots & c_{N-2} & 0 \\
0 & 0 & 0 & \cdots & 0 & c_{N-1}
\end{array}\right),
\end{gathered}
$$

where $\mu=(1 / 2 \Delta x), \eta=(1 / \operatorname{Re}), c_{k}=u_{k+1}-u_{k-1}$, and $k=$ $2, \ldots, N-2, c_{N-2}=-u_{N-2}$. EEI method and classical ERK method are applied to (21).

In Table 1, we compare the stability property of ERK method and EEI methods with $\mathrm{Re}=100$. The convergent steps with different spacial step are shown in Table 1. The convergent step lengths are obtained by computing three 


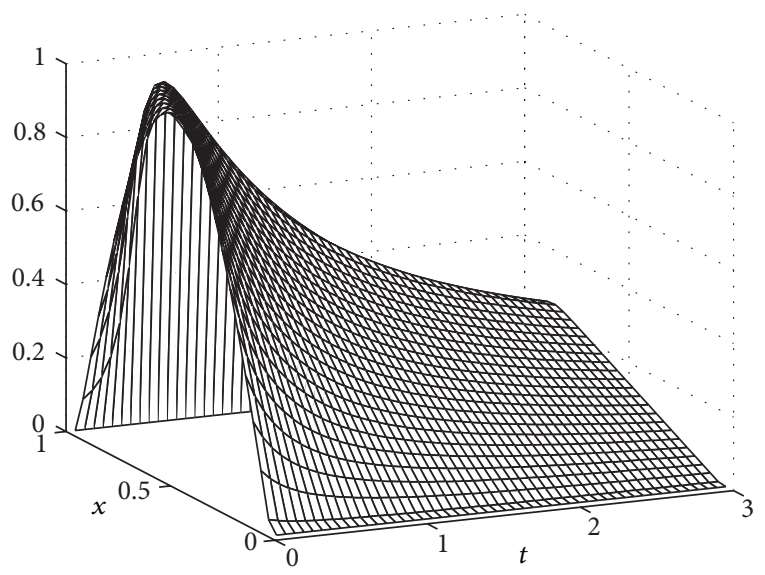

(a)

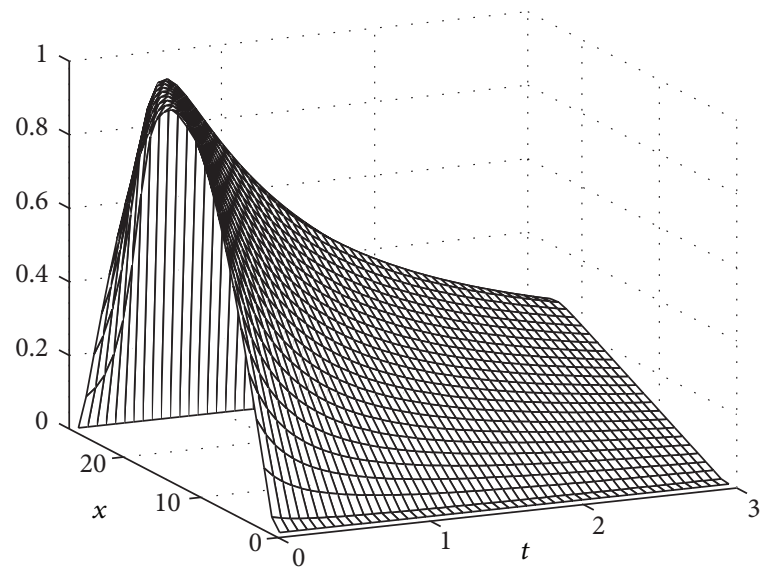

(b)

FIGURE 1: The numerical solutions of Burgers' equation at $t \in[0,3]$ obtained by ERK method and EEI method.

TABLE 1: The stability comparison of ERK method and EEI method.

\begin{tabular}{lcc}
\hline & ERK & EEI \\
\hline$N=50$ & 0.028 & 0.10 \\
$N=100$ & 0.007 & 0.080 \\
$N=200$ & 0.0016 & 0.025 \\
$N=400$ & 0.0005 & 0.0075 \\
\hline
\end{tabular}

TABLE 2: The errors of the numerical solutions and the exact solutions at different times by ERK method and EEI method.

\begin{tabular}{lcc}
\hline & ERK & EEI \\
\hline$t=1$ & $5.041 \times 10^{-5}$ & $5.044 \times 10^{-5}$ \\
$t=5$ & $8.765 \times 10^{-4}$ & $8.764 \times 10^{-4}$ \\
$t=10$ & $4.831 \times 10^{-3}$ & $4.831 \times 10^{-3}$ \\
$t=15$ & $1.265 \times 10^{-2}$ & $1.265 \times 10^{-2}$ \\
\hline
\end{tabular}

hundred steps to decide whether the step length is convergent. From Table 1, we can see that EEI method has better stability than ERK method. In Table 2, we compare the global errors of ERK method and EEI method with $N=100$ and $h=$ 0.002 . The global error is defined as $E_{r r}=\max \left|Y^{n}-Y(n \Delta t)\right|$, where $Y(n \Delta t)$ is the exact solution at $t=n \Delta t$. They have the same accuracy. In Figure 1, the numerical solutions of Burgers' equation at $t \in[0,3]$ are obtained by ERK method and the EEI method with $N=100, \operatorname{Re}=100$, and $h=0.005$. Figure 1(a) was obtained by ERK method and Figure 1(b) was obtained by EEI method. From Figure 1, we can get that they have the same accuracy.

\section{Numerical Experiments II: The Allen-Cahn Equation}

To the Allen-Cahn equation

$$
\frac{\partial u}{\partial t}=\varepsilon u_{x x}+u-u^{3}
$$

TABLE 3: The stability comparison of ERK method and EEI method.

\begin{tabular}{lcc}
\hline & ERK & EEI \\
\hline$N=50$ & 0.1 & 0.7 \\
$N=100$ & 0.02 & 0.25 \\
$N=200$ & 0.006 & 0.1 \\
$N=400$ & 0.0015 & 0.06 \\
\hline
\end{tabular}

with $\varepsilon=0.01$ and initial condition $u(x, 0)=0.53 x+$ $0.47 \sin (-1.5 \pi x)$. Suppose that $U=\left(u_{1}, u_{2}, \ldots, u_{N}\right)$, and (23) can be discretized into

$$
\begin{aligned}
& \frac{d U}{d t}=B(U) U \\
& B(U)=\frac{\varepsilon}{(\Delta x)^{2}}\left(\begin{array}{cccccc}
-2 & 1 & 0 & \cdots & 0 & 0 \\
1 & -2 & 1 & \cdots & 0 & 0 \\
\vdots & \vdots & \vdots & \ddots & \vdots & \vdots \\
0 & 0 & 0 & \cdots & -2 & 1 \\
0 & 0 & 0 & \cdots & 1 & -2
\end{array}\right) \\
& +\left(\begin{array}{cccccc}
c_{1} & 0 & 0 & \cdots & 0 & 0 \\
0 & c_{2} & 0 & \cdots & 0 & 0 \\
\vdots & \vdots & \vdots & \ddots & \vdots & \vdots \\
0 & 0 & 0 & \cdots & c_{N-1} & 0 \\
0 & 0 & 0 & \cdots & 0 & c_{N}
\end{array}\right),
\end{aligned}
$$

where $c_{k}=1-u_{k}^{2}$ and $k=1, \ldots, N$. EEI method and classical ERK method are applied to (24).

In Table 3, we compare the stability property of ERR method and EEI method by solving (24). When $N$ is different, the convergent step with different spacial step are shown in Table 3. The convergent step lengths are obtained by computing three hundred steps to decide whether the step length is convergent. From Table 3, we can see that the EEI method has better stability than ERK method. In Table 4, we 


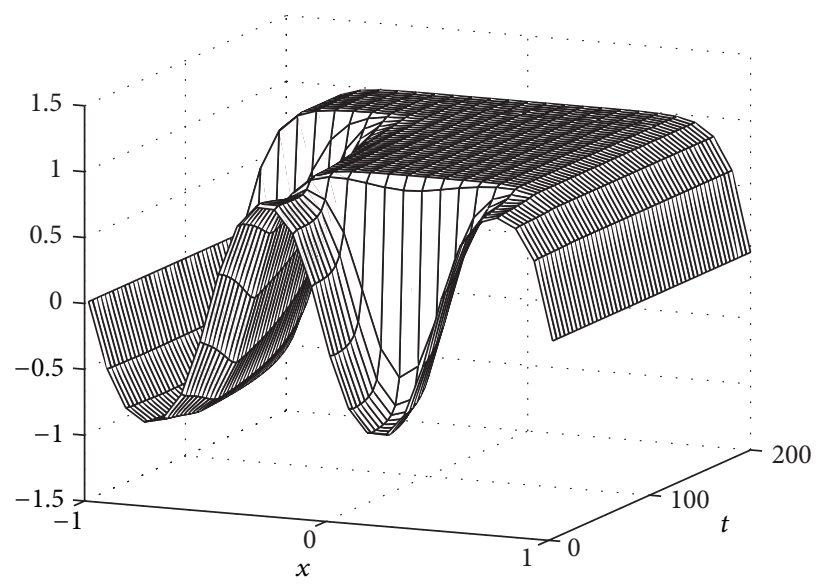

(a)

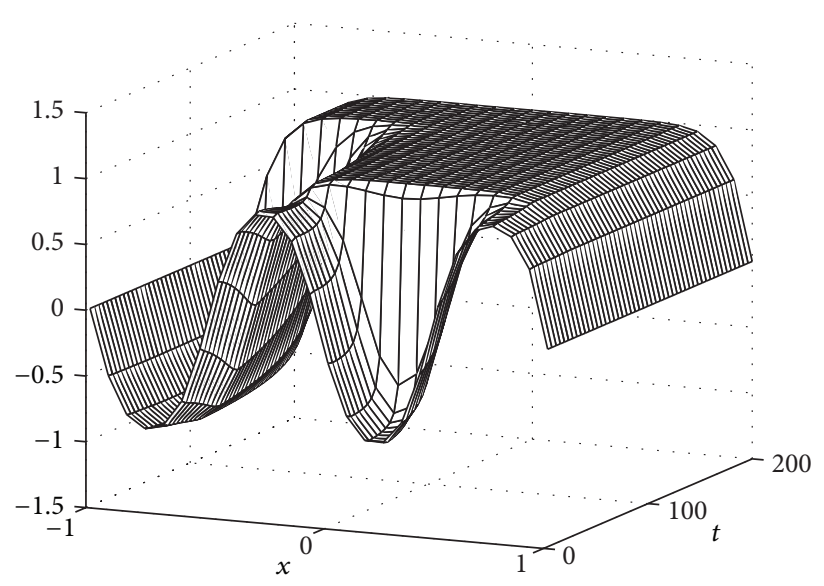

(b)

FIgURE 2: The numerical solutions of the Allen-Cahn equation at $t \in[0,200]$ obtained by the two methods with $N=100$ and $\varepsilon=0.01$.

TABLE 4: The errors of the numerical solutions at different times obtained by ERK method and EEI method.

\begin{tabular}{lccc}
\hline & ERK & EEI & Err \\
\hline$t=5$ & 0.50893803918253 & 0.50893804639290 & $10^{-8}$ \\
$t=10$ & 0.49120333210012 & 0.49120334154949 & $10^{-8}$ \\
$t=15$ & 0.46413587595252 & 0.46413588834075 & $10^{-8}$ \\
$t=20$ & 0.42975156048078 & 0.42975157699815 & $10^{-8}$ \\
\hline
\end{tabular}

compare the errors of the numerical solution at $x=-0.4$ with $h=0.01$ obtained by ERK method and the numerical solution obtained by EEI method. The error is up to $10^{-8}$ at different times; the error is $O\left(h^{4}\right)$. We can conclude that they have the same accuracy. In Figure 2, the numerical solutions of the Allen-Cahn equation in $t \in[0,200]$ were obtained by ERK method and EEI method with $N=100, h=0.01$, and $x=-0.4$. Figure 2(a) was obtained by ERK method. Figure 2(b) was obtained by EEI method. From Figure 2, we can get that the numerical results are the same. We can also conclude that the two methods have the same accuracy.

\section{Conclusions}

In this paper, the EEI method based on the idea of the Lie group method was proposed. The EEI method and the corresponding ERK method were applied to Burgers' equation and Allen-Cahn equation. Numerical results showed that the EEI method has better stability than the corresponding ERK methods. The two methods have the same accuracy. It is obvious that the explicit EER method is better than the ERK method in computing some stiff ordinary differential equations.

\section{Conflict of Interests}

The authors declare that there is no conflict of interests regarding the publication of this paper.

\section{Acknowledgment}

This work was supported by the National Natural Science Foundation of China (11161017 and 10905022).

\section{References}

[1] H. Munthe-Kaas, "Runge-Kutta methods on Lie groups," BIT Numerical Mathematics, vol. 38, no. 1, pp. 92-111, 1998.

[2] A. Iserles, H. Munthe-Kaas, S. P. Nørsett, and A. Zanna, "Lie group methods," Acta Numerica, vol. 9, pp. 215-365, 2000.

[3] G. J. Cooper, "Stability of Runge-Kutta methods for trajectory problems," IMA Journal of Numerical Analysis, vol. 7, no. 1, pp. $1-13,1987$.

[4] P. E. Crouch and R. Grossman, "Numerical integration of ordinary differential equations on manifolds," Journal of Nonlinear Science, vol. 3, no. 1, pp. 1-33, 1993.

[5] A. Iserles, "Solving linear ordinary differential equations by exponentials of iterated commutators," Numerische Mathematik, vol. 45, no. 2, pp. 183-199, 1984.

[6] M. P. Calvo, A. Iserles, and A. Zanna, "Conservative methods for the Toda lattice equations," IMA Journal of Numerical Analysis, vol. 19, no. 4, pp. 509-523, 1999.

[7] S. M. Cox and P. C. Matthews, "Exponential time differencing for stiff systems," Journal of Computational Physics, vol. 176, no. 2, pp. 430-455, 2002.

[8] L. Q. Chen, C. Wolverton, V. Vaithyanathan, and Z. Liu, "Modeling solid-state phase transformations and microstructure evolution," MRS Bulletion, vol. 26, pp. 197-292, 2001.

[9] G. Akrivis, M. Crouzeix, and C. Makridakis, "Implicitexplicit multistep methods for quasilinear parabolic equations," Numerische Mathematik, vol. 82, no. 4, pp. 521-541, 1999.

[10] E. Weinan, "Analysis of the heterogeneous multiscale method for ordinary differential equations," Communications in Mathematical Sciences, vol. 3, pp. 423-436, 2003.

[11] A. Zanna, "Collocation and relaxed collocation for the Fer and the Magnus expansions," SIAM Journal on Numerical Analysis, vol. 36, no. 4, pp. 1145-1182, 1999.

[12] L. R. Petzold, L. O. Jay, and J. Yen, "Numerical solution of highly oscillatory ordinary differential equations," Acta Numerica, vol. 6, pp. 437-483, 1997. 
[13] E. Hairer and G. Wanner, Solving Ordinary Differential Equations II: Stiff and Differential Algebraic Problems, Springer, New York, NY, USA, 1999.

[14] M. Z. Qin and Y. S. Wang, Structure-Preserving Algorithm for Partial Differential Equation, Zhejiang Science and Technology Publishing, 2012.

[15] R. C. Mittal and P. Singhal, "Numerical solution of Burger's equation," Communications in Numerical Methods in Engineering, vol. 9, pp. 163-175, 1993.

[16] S. Kutluay, A. R. Bahadir, and A. Özdeş, "Numerical solution of one-dimensional Burgers' equation: explicit and exactexplicit finite difference methods," Journal of Computational and Applied Mathematics, vol. 103, no. 2, pp. 251-261, 1999.

[17] E. R. Benton and G. W. Platzman, "A table of solution of the one-deimensional Burgers' equation," Quarterly of Applied Mathematics, vol. 30, pp. 195-212, 1972. 


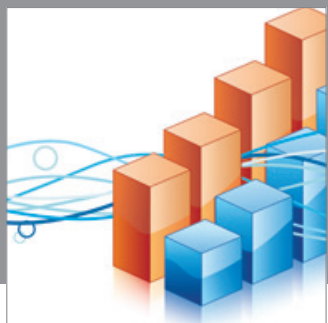

Advances in

Operations Research

mansans

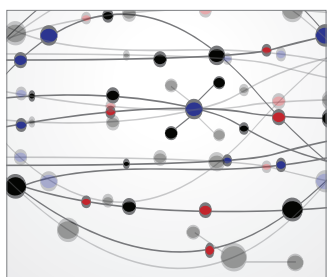

The Scientific World Journal
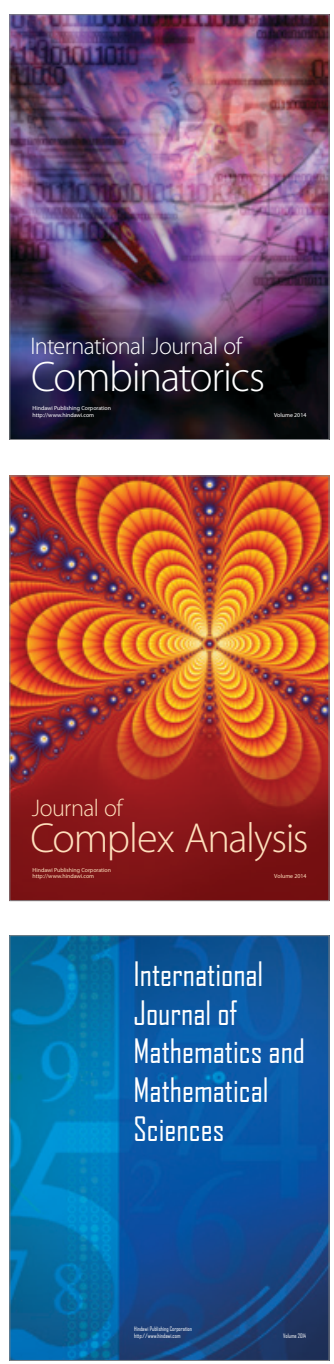
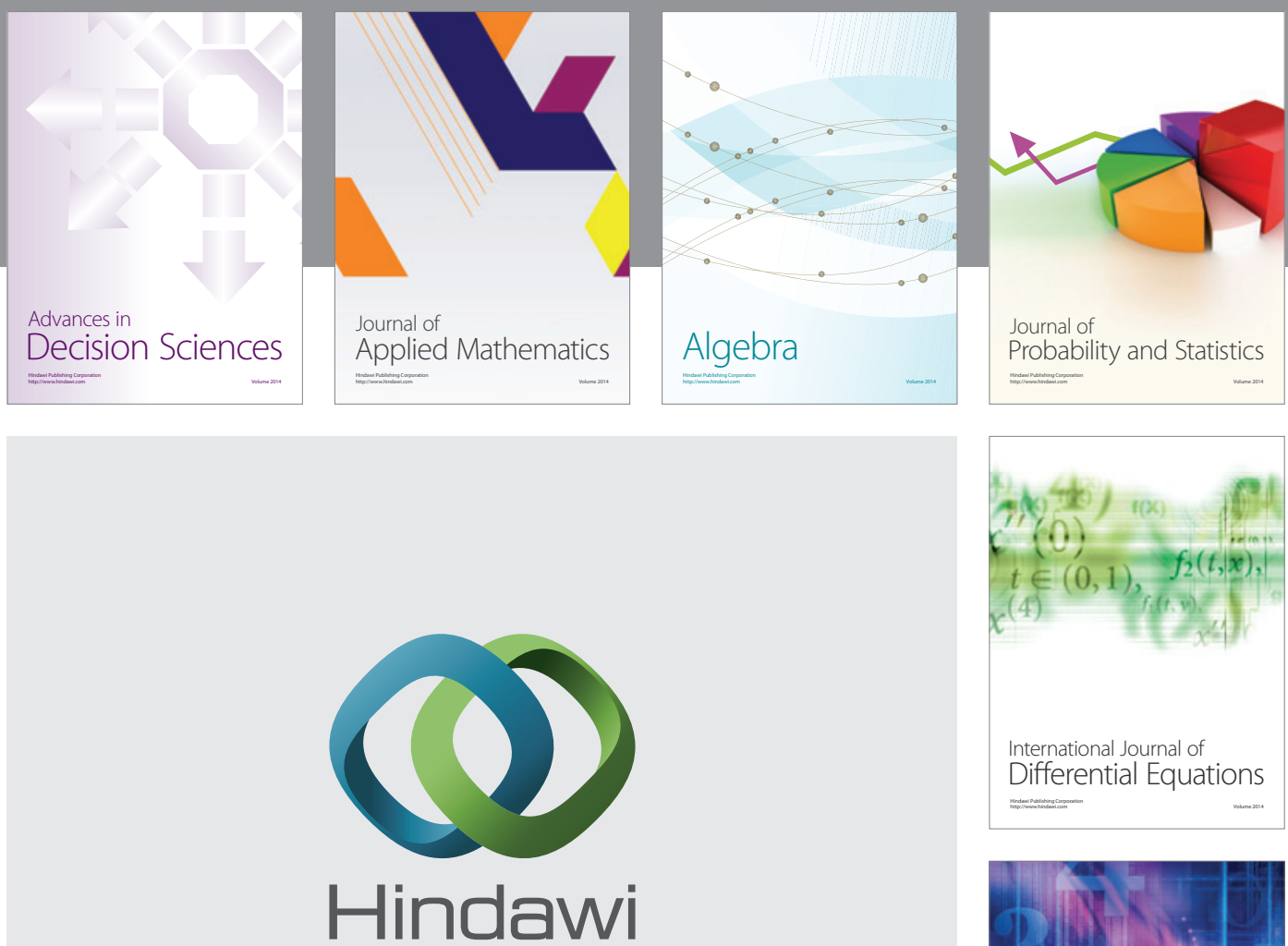

Submit your manuscripts at http://www.hindawi.com
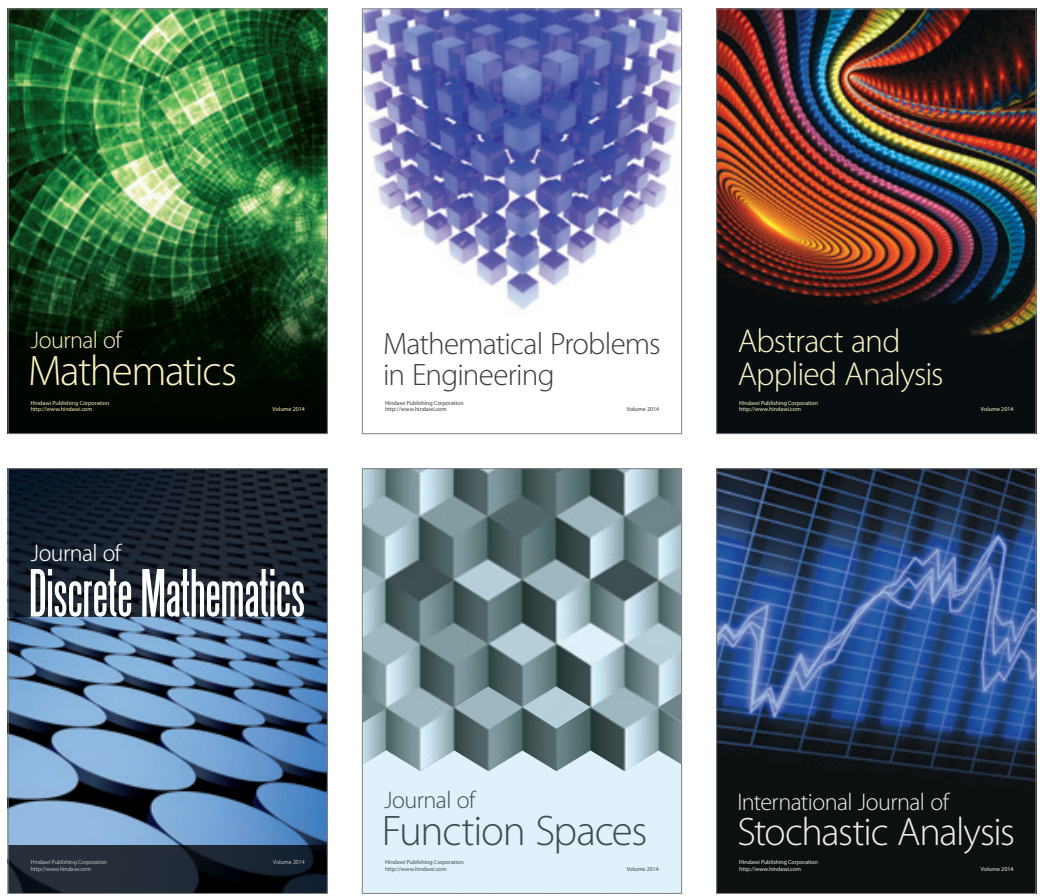

Journal of

Function Spaces

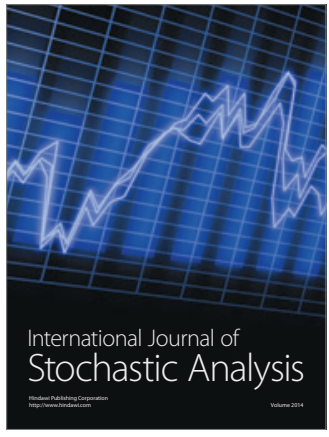

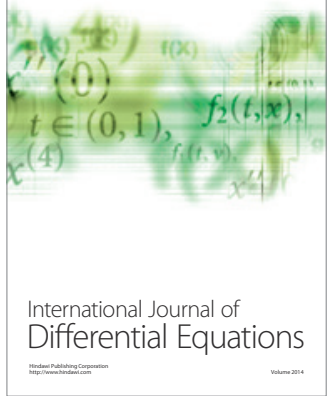
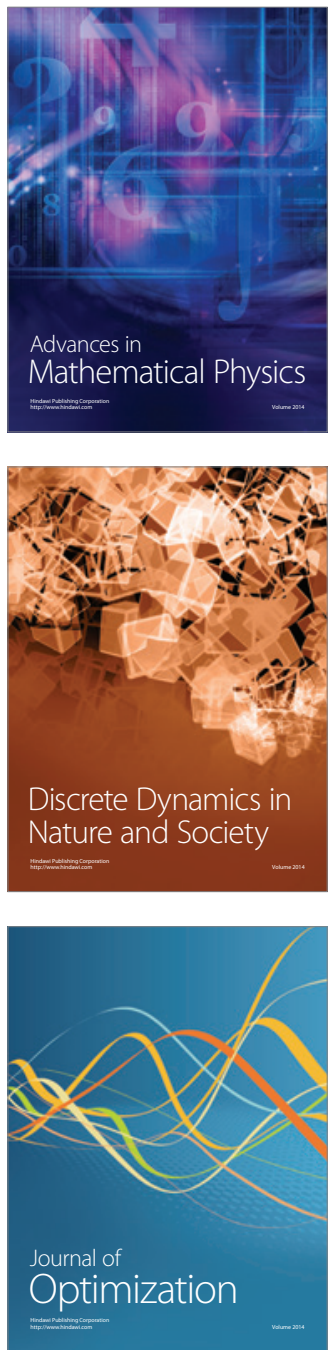